\title{
A General Study on the Transfer of Multimedia Messages over Mobile Adhoc Networks
}

\author{
K.Geetha \\ Associate Professor \\ Department of Comp.Sci \\ Periyar Arts College, \\ Cuddalore
}

\author{
N. Sreenath \\ Professor \\ Department of CSE \\ Pondicherry Engineering College \\ Puducherry
}

\begin{abstract}
Mobile Adhoc Networks are created and organized by a collection of nodes. Sending Multimedia data through these networks is a challenging process. In this paper some of the routing protocols namely AODV, DSDV, OLSR and FSR are taken to perform a general study. The QOS Parameters like Packet Delivery Ratio, Routing Control Overhead, Normalized Routing Load, Average End to End Delay, Throughput, and Jitter are analyzed for these protocols.
\end{abstract}

\section{General Terms}

Mobile Adhoc Networks.

\section{Keywords}

AODV, DSDV, OLSR, FSR, QOS Parameters.

\section{INTRODUCTION}

Multimedia is playing a vital role in almost all applications pertaining to government, education, finance etc. Nowadays, wireless networks especially, mobile adhoc networks are widely used for the transfer of data. While transferring multimedia data many parameters like delay, jitter which are related to the satisfaction of the user are to be considered. In this paper the transfer of multimedia data is assumed over mobile adhoc networks and the performance is analyzed using various parameters. In Mobile adhoc networking, two major types of protocols are used predominantly. They are Proactive routing protocols and reactive routing protocols. Although there exists many categories of protocols, these two classifications exists form the beginning of the wireless network. In Proactive routing, each node maintains the network topology information in the form of a table. Each node keeps the up-to-date information of the routes by constant updating of information. In reactive routing the route is created only when the source requests a route to a destination. The route is formed by the flooding technique. In this paper these two types of protocols are taken for analysis.

\section{OVERVIEW OF PROTOCOLS}

\subsection{AODV (Ad hoc On demand Distance Vector Protocol)}

AODV provides dynamic self starting routing between mobile nodes. It is a loop free protocol which uses destination sequence number for each route. It is a reactive routing protocol which selects route on demand. Route Requests (RREQS), Route Replies (RREPs) and Route Errors (RERRS) are the message types defined by AODV. The route is obtained when the destination is reached or an intermediate node with a route to destination is reached. When a link break is detected RERR message is used to notify other nodes that a loss has occurred. It deals with Route table management

\subsection{DSDV (Destination sequenced distance vector protocol)}

Each node maintains a routing table which stores, next hop, a sequence number and forwards the routing table to neighbors. It is a proactive routing protocol where the route is fixed initially. This guarantees loop free routes by proving a single path to destination. The path is selected by a distance vector shortest path algorithm. It introduces large overhead due to periodic update of messages.

\subsection{OLSR (Optimum Link State Routing protocol)}

It is a point to point proactive protocol, based on link state algorithm. Each node maintains topology information about the network by periodically exchanging link state messages. Here MPR (Multi point Relays) are used to transfer route information quickly. So routes between every node are available immediately when transmission begins

\subsection{FSR (Fish eye State Routing protocol)}

FSR is the Fish Eye State Routing protocol which exchanges the information to the neighbor nodes only and not with the entire network. This is a proactive routing protocol. The information in the table is maintained and corrected periodically. Entries corresponding to the nodes which are far away are propagated with less frequency than the nearby destinations. FSR produces the accurate distance and route information about the node which are within the scope of the source node. However, the knowledge of the best route to a distant destination is compensated by the fact that the route becomes progressively more accurate as the packet gets closer to destination

\section{PARAMETERS FOR MULTIMEDIA MESSAGES}

The performance metrics includes the following QoS parameters such as Packet Delivery Ratio, Routing Control Overhead, Normalized Routing Load, Average End to End delay, Throughput, and Jitter

\subsection{Pocket Delivery Ratio}

Packet delivery ratio(PDR) is defined as the ratio of the Number of Packets received to the Number of Packets Sent. This metric specifies the accuracy and reliability of the routing protocol. It will specify the loss rate of the protocols. It affects the maximum throughput that the network can support. 


\subsection{Routing Control Overhead}

Routing Control Over head is the number of Control packets used for sending the messages transmitted over the network, expressed in bits per second or packets per second This include requests, replies and error messages. It measures the scalability of the protocol, and the network

\subsection{Normalized Routing overload}

This is the ratio of the Number of control packets used to the Number of packets received at the destination. This gives a measure of the cost involved and the efficiency of the protocol.

\subsection{Average End to End Delay}

Average End to End delay is the average time taken by a data packet to reach from source node to destination node. It is the ratio of total delay to the number of packets received

\subsection{Throughput}

It is the number of bits received per second by the destination, which is the amount of data successfully transferred from source node to destination node

\subsection{Jitter}

Jitter is the delay between adjacent packets For multimedia message transfer. This is the main parameter taken into consideration for analysis

\section{SIMULATION AND ANALYSIS}

\subsection{Simulation}

The performance of the protocols AODV, DSDV, OLSR, and FSR are studied by sending multimedia messages over mobile adhoc networks using NS-2.

Table 1. Simulation Parameters

\begin{tabular}{|c|c|}
\hline Number of Nodes & 100 \\
\hline Simulation Area & $1000 \mathrm{mX} 1000 \mathrm{~m}$ \\
\hline Buffer Size (Queue Length) & 50 Pkts \\
\hline Packet size & 1024 Bytes \\
\hline Application Traffic & Video traffic(Evalvid) \\
\hline Simulation Time & $200 \mathrm{Secs}$ \\
\hline Number of Connections & 50 \\
\hline Connection duration $(\mathrm{sec})$ & 20 \\
\hline Data interval & $\begin{array}{l}0.01,0.02,0.03,0.04,0.05,0.06 \\
, 0.07,0.08,0.09,1.00\end{array}$ \\
\hline Connections & $\begin{array}{l}10,20,30,40,50,60,80,100,12 \\
0,140,150\end{array}$ \\
\hline
\end{tabular}

\subsection{Analysis}

The parameters are analyzed with the number of connections and the data interval

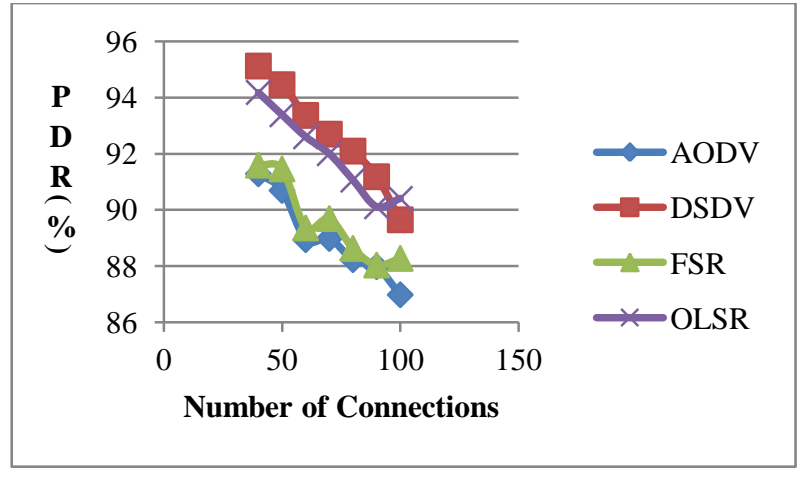

Figure 1. Packet Delivery Ratio with varying number of Connections

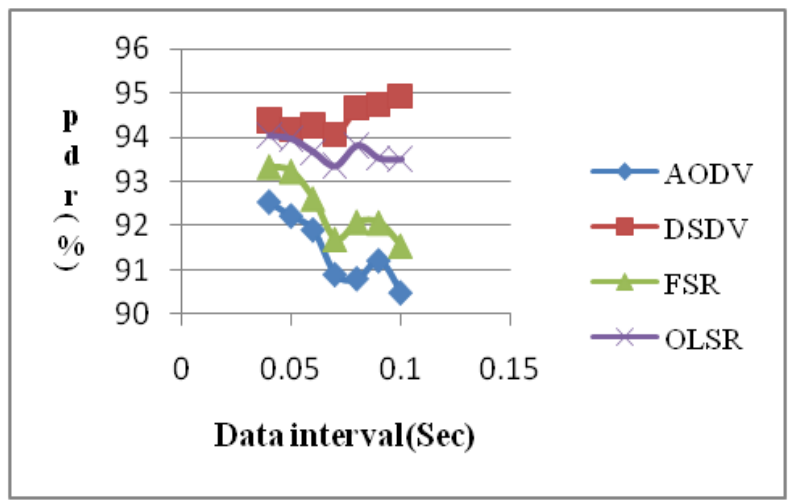

Figure 2. Packet Delivery Ratio with varying Data interval

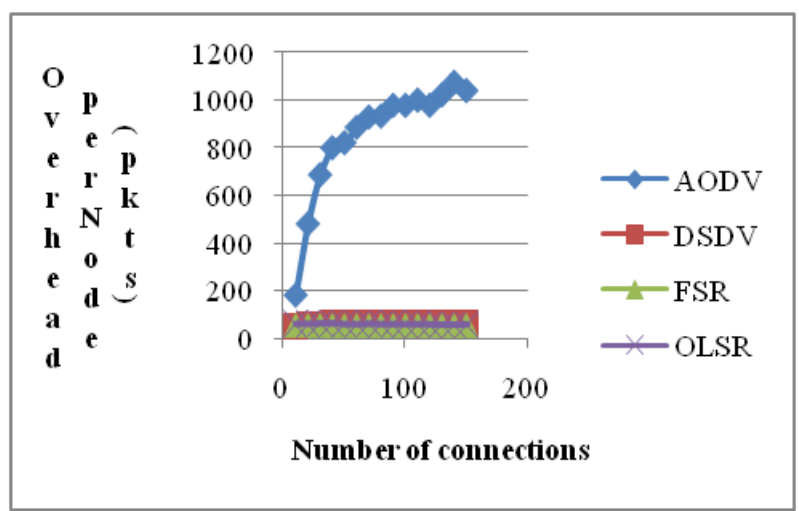

Figure 3. Overhead per node with varying number of connections

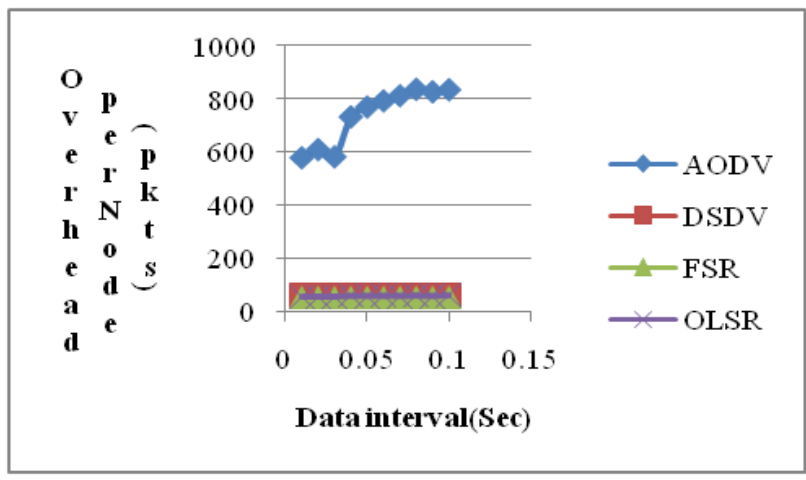

Figure 4. Overhead per Node with varying Data interval 


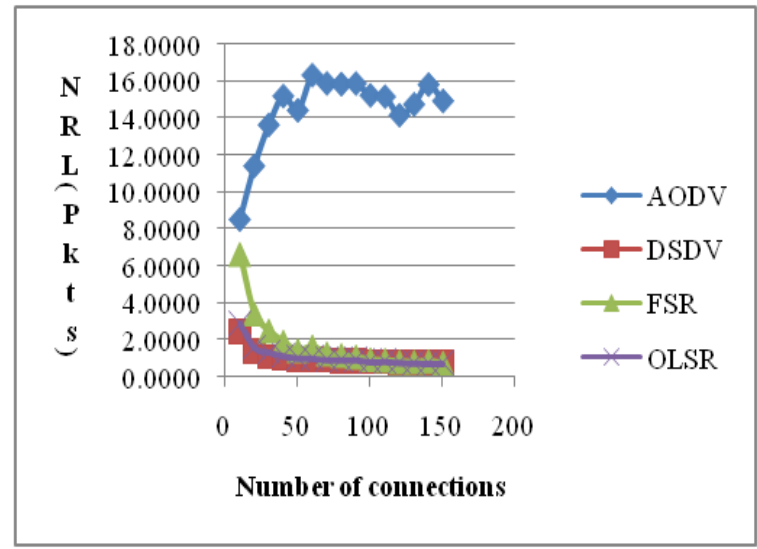

Figure 5 . NRL with varying Number of Connections

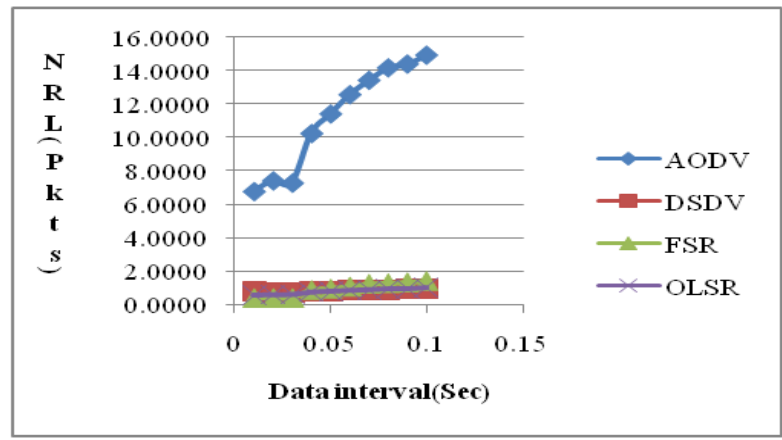

Figure 6. NRL with varying Data interval

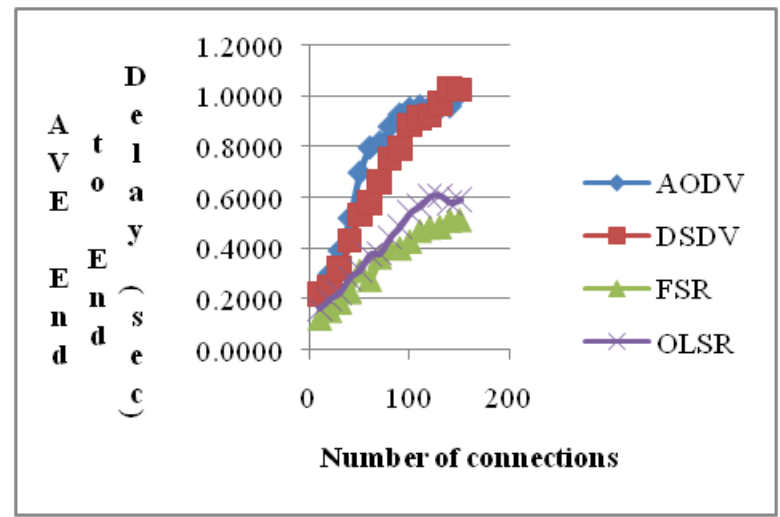

Figure 7. Average End to End Delay with varying Number of Connections

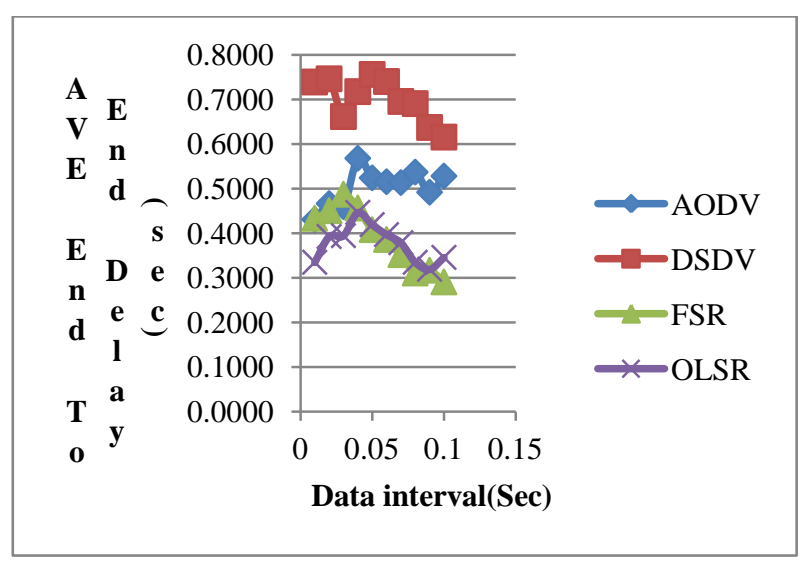

Figure 8. Average End to End Delay with varying Data Interval

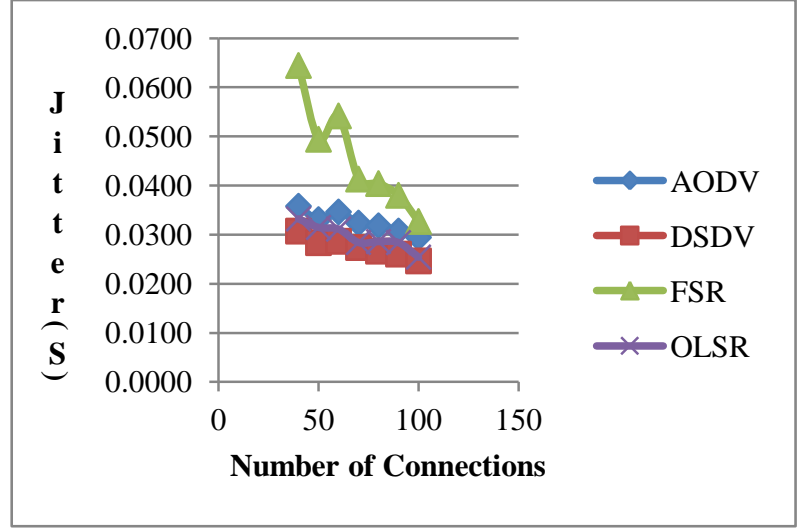

Figure 9. Jitter with varying number of Connections

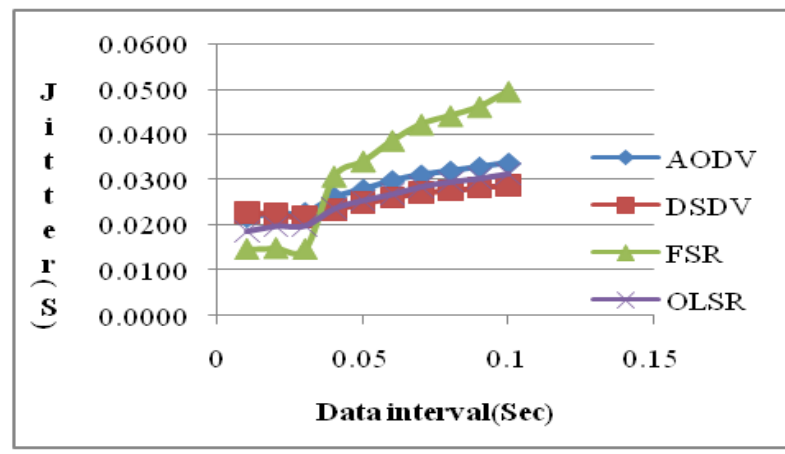

Figure 10. Jitter with varying Data Interval

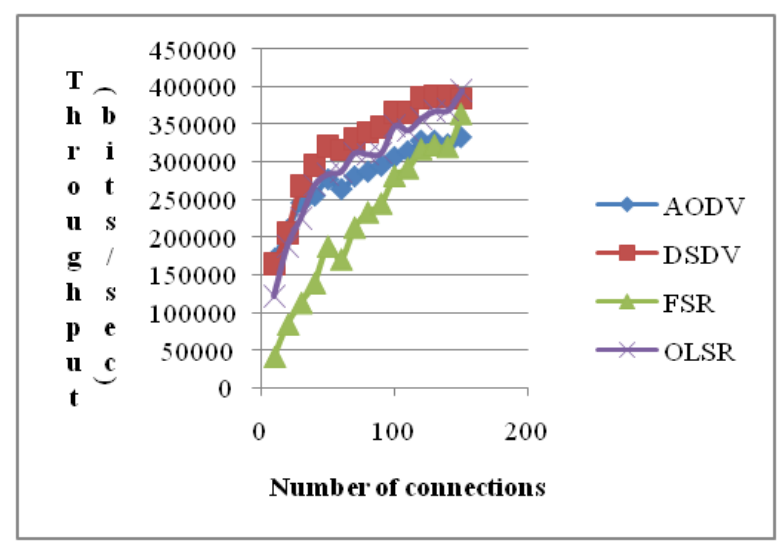

Figure 11. Throughput with varying number of connections

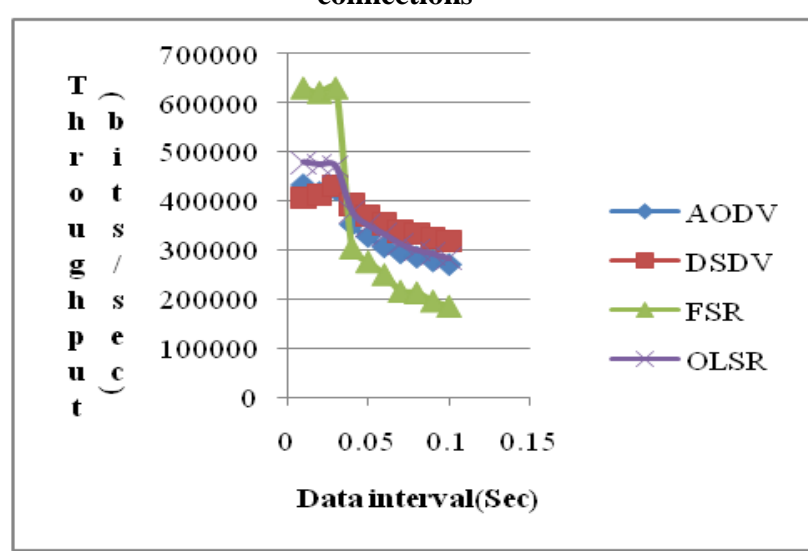

Figure 12.Throughput with varying Data interval 
The four protocols are behaving similarly with respect to PDR. In DSDV and OLSR protocol the Packet delivery ratio is Maximum. The overhead involved in AODV protocol is higher as it is a reactive routing protocol and needs often updating of the routing information. As the overhead increases the normalized routing load also increases. The AODV is having much higher NRL compared to other protocols since the number of control packets involved in the communication is higher. The OLSR is behaving well with respect to delay as it uses the technique of MPR (Multi Pont Relay). DSDV suffers from maximum End to End Delay. As the Route establishment is initiated after an initial delay for finding the best route to destination. The jitter is also low in the case of OLSR and DSDV protocols since the best route is fixed after running a best route finding algorithm. The throughput is good in case of OLSR and DSDV compared to the other two protocols when the first data packet comes, it is kept until the best route is found, thus avoiding the frequent changes of the route tables. Here, The rebroadcasts are reduced by delaying the advertisement of unstabilized routes. This enhances the accuracy of valid routes resulting in the increased throughput

\section{CONCLUSION}

Only the main QOS parameters are taken for analyzing the multimedia message transfer. Jitter and delay are the two main QOS parameters for multimedia message transfer. The Users satisfaction depends on the minimum jitter and minimum delay for multimedia messages. Our study shows that the DSDV and OLSR are the two protocols behaving well with respect to jitter and delay. No security aspects are considered in this paper. The message is assumed to be sent without any security threats. In future, these aspects may be considered and the analysis may be carried out.

\section{REFERENCES}

[1] Mehran Abolhasan Tadeusz Wysocki., Eryk Dutkiewicz "A review of routing protocols for mobile ad hoc networks " Elsevier journal of Ad hoc networks, pp. 122,2004 .

[2] C. Siva Ram Murthy \& B.S. Manoj, Ad-hoc Wireless networks, Pearson

[3] Loay Abusalah, Ashfaq Khokhar, and Mohsen Guizani "A Survey of Secure Mobile Ad Hoc Routing Protocols", IEEE communications and surveys, Tutorials Vol. 10, No. 4, Fourth Quarter 2008
[4] Al-Maashri. A and Ould Khaoua.M "Performance Analysis of MANET Routing protocols in the presence of Self similar traffic", Proceedings of the 31st IEEE Conference on Local Computer Networks, 2006, 14-16 November 2006, Tampa, Florida USA.

[5] C. Perkins, E. Belding-Royer, and S. Das, "Ad Hoc onDemand Distance Vector (AODV) Routing". IETF RFC3561, from http://www.ietf.org/rfc/rfc3561.txt.

[6] Rajneesh Kumar Gujral, Manpreet Singh, "Analyzing the Impact of Scalability on QoS-aware Routing for MANETs “ IJCSI International Journal of Computer Science Issues, Vol. 8, Issue 3, No. 1, May 2011 pp 487495

[7] S. Gowrishankar, T.G. Basavaraju, M. Singh, Subir Kumar Sarkar "Scenario based Performance Analysis of AODV and OLSR in Mobile Ad hoc Networks" Proceedings of the 24th South East Asia Regional Computer Conference, International Journal of the Computer, the Internet and Management,, November 1819, 2007, Bangkok, Thailand

[8] The Network Simulator, NS-2, from www.isi.edu/nsnam/ns, 2006

[9] S. R. Das, C. E. Perkins, E. M. Royer and M. K. Marina," Performance Comparison of Two On-demand Routing Protocols for Ad Hoc Networks," in IEEE Personal Communications magazine, special issue on Mobile Ad Hoc Networks, Vol. 8, No. 1, pp. 16-29, Feb 2001

[10] P. Jacquet, P. Muhlethaler, T. Clausen, A. Laouiti, A. Qayyum, L. Viennot " Optimized Link State Routing Protocol for Adhoc networks " proceedings of the IEEE Multitopic Conference INMIC 2001 pp 62-68

[11] Guangyu Pei Mario Gerla Tsu-Wei Chen Fisheye State Routing: A Routing Scheme for Ad Hoc Wireless" proceedings of the IEEE International Conference June 2000 pp 70-74

[12] N. Javaid, M. Yousaf A. Ahmad, A. Naveed, K. Djouani" Evaluating Impact of Mobility on Wireless Routing Protocols" proceedings of the IEEE Symposium on Wireless Technology and Applications (ISWTA) 25 28 Langkawi, Malaysia September 2011 